\title{
Mps1 Inhibitor BAY 1217389
}

National Cancer Institute

\section{Source}

National Cancer Institute. Mps1 Inhibitor BAY 1217389. NCI Thesaurus. Code C122707.

An orally bioavailable, selective inhibitor of the serine/threonine kinase monopolar spindle 1 (Mps1, TTK), with potential antineoplastic activity. Upon administration, the Mps1 inhibitor BAY 1217389 selectively binds to and inhibits the activity of Mps1. This inactivates the spindle assembly checkpoint (SAC), accelerates mitosis, causes chromosomal misalignment and missegregation, and mitotic checkpoint complex destabilization. This induces cell death in Mps1-overexpressing cancer cells. Mps1, a kinase expressed in proliferating normal tissues and aberrantly overexpressed in a wide range of human tumors, is activated during mitosis and is essential for proper SAC functioning and chromosome alignment. 